SCHweISFURTH: Ohne weitere Literaturkenntnisse möchte ich doch meinen, daß es mit Hilfe von kontinuierlichen Kulturverfahren möglich sein sollte, konstante Mischkulturen zu erhalten. Wie schon gesagt, wir haben in Homburg Untersuchungen in dieser Richtung begonnen.

\title{
Vermehrung von Mikroorganismen in Öltropfen
}

Schwersfurth: Ich möchte über erste Versuche zur Frage der Vermehrung von Mikroorganismen in Oltropfen berichten. Bei Versuchen über den mikrobiellen Abbau von Kohlenwasserstoffen (KWS) in von unten belüfteten Kolben fiel als erstes auf, daß nach 2 bis 3 Tagen der Kultur eine Emulgierung stattfindet, bei der anfangs größere Tropfen des jeweils zugefügten Substrates vorlagen, die in der Folge mechanisch, durch vermutlich gebildete oberflächenaktive Stoffe und durch Verzehr weiter verkleinert wurden. Mit zunehmendem Alter der Mischkulturen und der Erschöpfung der $\mathrm{N}$ - und $\mathrm{PO}_{4}$-Quellen war der $\mathrm{KWS}$ soweit verändert, daß nach Abstellen der Belüftung Rest-Kohlenwasserstoffe teilweise absanken und am Boden liegenblieben.

Da beim Durchströmen der beimpften Flüssigkeitskulturen mit Stickstoff die Bildung von KWS-Tropfen nicht stattfand, eine rein mechanische Zerstörung der auf die Oberfläche der Mineralsalzlösung gegebenen Kohlenwasserstoffe also ausblieb, muß eine Beteiligung von Stoffwechselprodukten der Mikroorganismen vorliegen. Beobachtungen hierzu sind bereits verschiedentlich publiziert worden (LA Rrvière 1955a, b).

Die Tropfenbildung veranlaßte uns zu mikroskopischen Untersuchungen, da es auch von grundsätzlichem Interesse war, festzustellen, ob Mikroben in Oltropfen eindringen können oder ob ein Angriff nur von außen erfolgen kann.

Beim Abbau von $\mathrm{C}_{16}$-Paraffin fiel nach etwa zweitägiger Kulturdauer auf, daß auf den KWSTropfen kleine Tröpfchen lagen. In der gleichen Kultur fanden sich nach 3 bis 4 Tagen massenhaft Oltropfen, deren Oberfläche dicht mit Bakterien besetzt waren. Später traten dann die kugelrunden Oltropfen zurück, es zeigten sich schlierenförmige Gebilde, die nach weiterer Bebrütung zu „Detritus“ zerfielen. In gealterten Flüssigkeitskulturen lagen die Mikroorganismen frei in der Lösung und am Boden vor.

Im Gegensatz hierzu schien nach den ersten Versuchen mit Rohöl ein Eindringen der Bakterien in das OOl relativ schnell zu erfolgen. Ein Beweis für das Eindringen konnte bislang höchstens indirekt geführt werden, da bei der mikroskopischen Untersuchung besiedelten Öls ohne besondere Kunstgriffe nicht schlüssig beurteilt werden konnte, ob die Mikroben nicht doch noch auf der Oberfläche von Olschlieren oder -tropfen lagen.

Bei Kulturen mit Rohöl fiel auf, daß fast alle Bakterien in (oder auf?) den Olschlieren lagen und daß sie auch bei gealterten Kulturen im Bodensatz nur selten frei in der Lösung zu finden waren. Dies könnte für eine Vermehrung der Mikroben im Ol selbst sprechen. Ferner beobachteten wir bei Objektträgerkulturen, daß Öltropfen mit Bakterien innerhalb weniger Sekunden „in“ steriles OOl eindringen und sich die Bakterien in der Folge darin vermehren können.

WaLLHäUsser: Was sollen die Bakterien ausrichten können, die in den Öltropfen eingedrungen sind?

GUNKEL: Heyer (1966) berichtete über das Wachstum von Bakterien in den wäßrigen Medien. Er ist der Ansicht, daß der Ubertritt in das Ol ein rein physikalischer Vorgang ist, der nicht an die lebende Zelle gebunden ist. Verantwortlich soll ein Uberwiegen hydrophober Gruppen auf der Zelloberfläche sein. Eine Vermehrung soll auch hier fast ausschließlich an der Grenzfläche Öl-Wasser stattfinden, wobei hier die Bakterien die Olseite der Grenzfläche besiedeln.

\section{Isolation von Reinkulturen ölabbauender Bakterien}

THoN: Ich möchte über eine Methode berichten, die auf relativ einfache Weise gestattet, Reinkulturen ölabbauender Mikroorganismen zu isolieren. Hiermit kann man Ölabbauer auch aus Untersuchungsmaterial gewinnen, in welchem sie in sehr geringer Anzahl vorkommen, ohne 